\title{
Transgenic expression of the deoxynucleotide carrier causes mitochondrial damage that is enhanced by NRTIs for AIDS
}

\author{
William Lewis ${ }^{1}$, Chad P Haase ${ }^{1}$, Yoon K Miller ${ }^{1}$, Brandy Ferguson ${ }^{1}$, Tami Stuart ${ }^{1}$, \\ Tomika Ludaway ${ }^{1}$, Jamie McNaught ${ }^{1}$, Rodney Russ ${ }^{1}$, Jeffrey Steltzer ${ }^{1}$, Robert Santoianni ${ }^{1}$, \\ Robert Long ${ }^{2}$, Giuseppe Fiermonte ${ }^{3}$ and Ferdinando Palmieri ${ }^{3}$ \\ ${ }^{1}$ Department of Pathology, Emory University School of Medicine, Atlanta, GA, USA; ${ }^{2}$ Radiological Sciences, \\ Emory University School of Medicine, Atlanta, GA, USA and ${ }^{3}$ Department of Pharmaco-Biology, University \\ of Bari, Bari, Italy
}

\begin{abstract}
Nucleoside reverse transcriptase inhibitors (NRTIs) are antiretrovirals for AIDS with limiting mitochondrial side effects. The mitochondrial deoxynucleotide carrier (DNC) transports phosphorylated nucleosides for mitochondrial DNA replication and can transport phosphorylated NRTIs into mitochondria. Transgenic mice (TG) that exclusively overexpress DNC in the heart tested DNC's role in mitochondrial dysfunction from NRTIs. Two TG lines were created that overexpressed the human DNC gene in murine myocardium. Cardiac and mitochondrial structure and function were examined by magnetic resonance imaging, echocardiography, electrocardiography, transmission electron microscopy, and plasma lactate. Antiretroviral combinations (HAART) that contained NRTIs (stavudine $\left(2^{\prime}, 3^{\prime}\right.$-didehydro-2', 3'-deoxythymidine or d4T)/lamivudine/indinavir; or zidovudine ( $3^{\prime}$ azido-3'-deoxythymidine or AZT)/lamivudine/indinavir; 35 days) were administered to simulate AIDS therapy. In parallel, a HAART combination without NRTIs (nevirapine/efavirenz/indinavir; 35 days) served as an NRTI-sparing, control regimen. Untreated DNC TGs exhibited normal cardiac function but abnormal mitochondrial ultrastructure. HAART that contained NRTIs caused cardiomyopathy in TGs with increased left ventricle mass and volume, heart rate variability, and worse mitochondrial ultrastructural defects. In contrast, treatment with an NRTI-sparing HAART regimen caused no cardiac changes. Data suggest the DNC is integral to mitochondrial homeostasis in vivo and may relate mechanistically to mitochondrial dysfunction in patients treated with HAART regimens that contain NRTIs.
\end{abstract}

Laboratory Investigation (2005) 85, 972-981. doi:10.1038/labinvest.3700301; published online 13 June 2005

Keywords: deoxynucleotide; NRTI; AIDS; antiretroviral; mitochondrial import; DNC; cardiac; HIV

The inner mitochondrial membrane contains transport proteins to move molecules into and out of the matrix. Members of the mitochondrial carrier family of proteins contain three conserved tandemrepeated sequences ( $\sim 100$ residues with two hydrophobic transmembrane $\alpha$-helices and a hydrophilic segment thought to be an extramembranous loop ${ }^{1}$ ). One of these proteins functions as a deoxynucleotide carrier (DNC) to import phosphorylated precursors of mitochondrial (mt-) DNA synthesis and has been characterized. ${ }^{1-3}$ Toxicity to mitochondria from antiretroviral nucleoside reverse transcriptase

Correspondence: Dr W Lewis, MD, Department of Pathology, Emory University School of Medicine, 7117 Woodruff Memorial Building, 101 Woodruff Circle, Atlanta, GA 30322, USA.

E-mail: wlewis@emory.edu

Received 24 December 2004; revised 29 March 2005; accepted 5 April 2005; published online 13 June 2005 inhibitors (NRTI) is an established side effect of AIDS therapy that limits effective treatment (reviewed in Lewis et $a l^{4}$ ). Alternative combinations that are NRTI-sparing may be effective if toxicity is a significant clinical problem (reviewed in Joly et $a l^{5}$ ).

Since DNC provides a route for mitochondrial uptake of NRTIs including zidovudine $\left(3^{\prime}\right.$ azido- $3^{\prime}-$ deoxythymidine or AZT) and stavudine $\left(2^{\prime}, 3^{\prime}-\right.$ didehydro-2', 3'-deoxythymidine or $\mathrm{d} 4 \mathrm{~T}$ ), its role in mitochondrial NRTI import and toxicity was addressed in vivo using transgenic mice (TG) and HAART treatment. One HAART regimen included NRTIs (with either an AZT or D4T 'backbone'). A second NRTI-sparing regimen was administered in parallel as a control.

DNC overexpressed in the murine heart caused reduplicated mitochondrial cristae, but not cardiac dysfunction. The addition of therapeutic doses of HAART that contained NRTIs worsened cardiac 
mitochondrial destruction and caused cardiomyopathy (CM) with cardiac dysfunction. In contrast, treatment with NRTI-sparing HAART caused no cardiac changes. Data from DNC TGs indicate that DNC plays a critical role in nucleotide homeostasis, pharmacological handling of NRTIs, and in the development of mitochondrial side effects from NRTI-containing HAART regimens.

\section{Materials and methods}

\section{Generation of $\alpha$-MyHC/DNC TG}

Established methods were employed essentially as described previously. ${ }^{6}$ A 963-bp fragment containing the human DNC gene was amplified using the primers $\mathrm{DNC5}^{\prime}$ and $\mathrm{DNC}^{\prime}$ and cloned into a pBluescript $5 \mathrm{~K}$ vector. The $\alpha$-MyHC clone 26 (compliments of Jeff Robbins, Children's Research Foundation, Cincinnati, OH, USA ${ }^{7}$ ) was digested with SalI and HindIII (Roche Applied Science, Indianapolis, IN, USA) to facilitate construction of the final vector.

\section{Treatment Protocols}

Procedures complied with Emory IACUC and NIH guidelines. Drugs were from the manufacturers or from the Emory Center for AIDS Research Pharmacology Core (Raymond Schinazi, VA Medical Center, Decatur, GA, USA). Dosing was carried out by daily gavage (morning) at doses that resemble human therapy. Doses used were $\mathrm{AZT}=0.22 \mathrm{mg} /$ day; $\mathrm{D} 4 \mathrm{~T}=0.0285 \mathrm{mg} / \mathrm{day} ; \quad 3 \mathrm{TC}=0.11 \mathrm{mg} / \mathrm{day} ; \quad$ and indinavir $=0.9 \mathrm{mg} /$ day. For protocols with NRTIsparing HAART, cohorts received a HAART combination without NRTIs (nevirapine $=0.14 \mathrm{mg}$ /day; efavirenz $=0.21 \mathrm{mg} /$ day; $\quad$ indinavir $=0.9 \mathrm{mg} /$ day). Drug-free vehicle served as control. A $1 \mathrm{ml}$ syringe was fitted with a 20-Gauge $5 \mathrm{~cm}$ curved feeding needle with a $2.25 \mathrm{~mm}$ ball at the end. The tip was inserted down the esophagus and medication administered $(250 \mu \mathrm{l})$.

\section{Genotyping}

For the TG line $\alpha$-MyHC/DNC, the transgene was detected in the founders and their offspring using Southern blotting and PCR essentially as described previously. ${ }^{6}$

\section{RNA Extraction, Northern Analysis, and Immunoblotting}

Methods resembled those used by us. ${ }^{6}$

\section{Echocardiography (ECHO) in DNC TG and WT}

For ECHO observations in the HAART protocols with AZT backbone, TG + AZT HAART $N=9$;
TG + vehicle $N=7 ;$ WT + AZT HAART $N=7$; and WT + vehicle $N=5$. For HAART protocols containing D4T, TG + D4T HAART $N=10$; $\mathrm{TG}+$ vehicle $N=10$; WT + D4T HAART $N=12$; and WT + vehicle $N=11$. For NRTI-sparing HAART, TG + HAART $N=8 ; \quad$ TG + vehicle $N=7 ; \quad$ WT + HAART $\quad N=9$; HAART $N=12$; and WT + vehicle $N=9$. ECHO studies were performed in age- and gender-matched (littermate) WT and TGs. Methods were those described previously. ${ }^{8}$

\section{Magnetic Resonance Imaging (MRI) Protocol}

Mice from the AZT-containing HAART protocol were used for MRI evaluation. Mice were sedated using ketamine/xylazine and kept warm under a warming light. To maintain body temperature, a continuously circulating water-jacketed platform was used. After sedation, a small animal monitoring system was employed. Two platinum electrocardiography (ECG) electrodes were in the forelimb and hind limb. A rectal probe monitored core temperature. A computer was used to monitor continuous real-time waveforms, measured values, trends, and gating pulses. Data acquisition was controlled by menu-driven software. Body temperature was monitored throughout and maintained at $37 \pm 1^{\circ} \mathrm{C}$. The animal was positioned inside a $3.7 \mathrm{~cm}$ (internal diameter) 16-element 'birdcage' body coil, which was inserted to the magnet center. The ECG signal triggered the MR scanner. Imaging was performed on a horizontal bore, Varian Inova 4.7 T MRI scanner with an insert gradient coil $24 \mathrm{G} / \mathrm{cm}$ and $250 \mu$ s rise time. ECG-gated survey images were acquired in the coronal plane to locate the heart. Planing of a slice along the long axis of the heart was performed from base to apex. This slice was acquired with a 2dimension, cine FLASH sequence. Planing was performed from a set of contiguous images on the VLA image at end-diastole, 1-mm thick, short-axis images that were acquired using the same 2-dimension, 10-frame cine FLASH sequence used for the VLA images. An echo time of $3.2 \mathrm{~ms}$ and repetition times of 100-150 ms (depending on heart rate (HR)) was used. An $8 \mathrm{~cm} \times 4 \mathrm{~cm}$ field of view was acquired with a matrix size of $256 \times 128$, resulting in a final resolution of $312 \times 312 \times 1000 \mu$. To improve signalto-noise ratio, signals were averaged. Acquisition time for each slice was $\leq 2 \mathrm{~min}$. Six to eight $1-\mathrm{mm}$ thick short-axis slices covered the heart.

\section{Pathological Evaluations with Transmission Electron Microscopy (EM) and Morphometric Analysis of Mitochondrial Damage}

Ultrastructure $(N=12 ; 8-12$ weeks $)$ was evaluated using transmission EM. Sections (approximately $1 \mathrm{~mm}$ cubes) were rapidly fixed in diluted Karnovsky's fixative as we have carried out in the past and processed for EM. ${ }^{8}$ Transmission EM sections were 
viewed on a JEOL 100CX microscope (JEOLCO, Tokyo) and evaluated by blinded observers. Electron photomicrographs were taken. Representative highpower views were included as demonstration of ultrastructural abnormalities.

Quantitative analysis of mitochondrial damage was performed essentially as we have carried out in the past. $^{9}$ Photomicrographs were enlarged to $8 \times 10$ inch prints. Each electron photomicrograph was reviewed independently by two investigators for the presence of structurally abnormal mitochondria, for the presence of intramitochondrial lamellar bodies, and for intramitochondrial paracrystals as reported in human AZT-induced skeletal muscle mitochondrial myopathy. ${ }^{10}$ Structurally abnormal mitochondria were operationally defined as having loss or dissolution of $\geq 25 \%$ of cristae. The number of damaged mitochondria per photomicrograph was quantified and the fraction of mitochondria damaged was defined and analyzed by ANOVA.

\section{Real-Time ECG in TGs}

For ECG data from the HAART protocols with an AZT backbone, TG + AZT HAART $N=11$; TG + vehicle $N=8$; WT + AZT HAART $N=8$; and WT + vehicle $N=10$. For HAART containing D4T, TG + D4T HAART $N=5 ; \quad$ TG + vehicle $N=6$; $\mathrm{WT}+\mathrm{D} 4 \mathrm{~T}$ HAART $N=6$; and WT + vehicle $N=5$. For NRTI-sparing HAART, TG + HAART $N=6$; TG + vehicle $\quad N=6 ; \quad$ WT + HAART $N=8 ; \quad$ and $\mathrm{WT}+$ vehicle $N=4$. Methods employed resemble those used by us previously. ${ }^{11}$ Data from continuous recordings of at least 25 ECG signals were used for analyses and interpretation.

\section{ECG Analyses}

DNC cohorts ( $N=5-11 ; 25-55$ signals per cohort) were evaluated using real-time ECGs (without confounding effects of anesthesia). Each signal was analyzed using e-MOUSE, Internet-based physiologic waveform analyses software as described. ${ }^{12,13}$ The program used Fourier analyses and linear timeinvariant digital filtering of frequencies below $2 \mathrm{~Hz}$ and above $100 \mathrm{~Hz}$ to minimize noise. Software uses a peak detection algorithm to find the peak of the $\mathrm{R}$ waves and to calculate HR. The inverted and/or biphasic portions of the $\mathrm{T}$ wave were used to calculate the $\mathrm{Q}-\mathrm{T}$ interval, ${ }^{14}$ which was rate corrected (Q-Tc) by an algorithm developed for mice. Spurious data resulting from unfiltered noise or motion artifacts was rejected. The mean of the ECG time intervals for each set of waveforms was calculated. HR variability (HRV) was calculated as the standard deviation of all R-R intervals for each set of ECG signals, and the coefficient of variation (\%) was calculated as the ratio of mean HRV to mean HR. ${ }^{15}$

\section{Plasma Lactate}

At termination of the experiments, samples were obtained by retro-orbital bleeding of anesthetized TGs and WTs $(N=4-12)$ in ways that resemble those used by us previously. ${ }^{8}$ For lactate measurements on HAART protocol, TG + AZT HAART $N=6$; TG + vehicle $N=6$; WT + AZT HAART $N=10 ;$ and WT + vehicle $N=9$. For HAART containing D4T, $\mathrm{TG}+\mathrm{D} 4 \mathrm{~T} \quad$ HAART $\quad N=9 ; \quad \mathrm{TG}+$ vehicle $\quad N=9$; $\mathrm{WT}+\mathrm{D} 4 \mathrm{~T}$ HAART $N=12 ;$ and $\mathrm{WT}+$ vehicle $N=11$. For NRTI-sparing HAART, TG + HAART $N=8$; TG + vehicle $N=7$; WT + HAART $N=9$; and WT + vehicle $N=9$. Plasma was separated, stored briefly at $4{ }^{\circ} \mathrm{C}$, and aliquots $(10 \mu \mathrm{l})$ were analyzed for lactate colorimetrically using a commercial kit (Sigma, St Louis, MO, USA). Assays were performed on a Versamax ${ }^{\circledR}$ microtiter plate reader (Molecular Devices, Sunnyvale, CA, USA) and run in triplicate with external standards with each run.

\section{Statistical Analysis}

Determinations from all groups were compared by ANOVA as we have carried out in the past. ${ }^{8}$

\section{Results}

\section{General}

Growth, maturation, and fertility were similar in TG and WT.

\section{Targeted Transgenesis of DNC}

Two hemizygous TG lines were created. DNC RNA was abundant in myocardium of TGs. WT littermates exhibited native DNC expression. DNC RNA abundance was low in other tissues and followed the reported distribution ${ }^{2}$ (Figure 1a). DNC polyA ${ }^{+}$ RNA from TG myocardium indicated significant DNC mRNA present in the heart (Figure 1b). Immunoblot of mitochondrial extracts from hearts revealed abundant DNC in extracts from TG hearts compared to those of WT hearts (Figure 1c).

\section{ECHO and MRI Data from TGs and HAART Treatment}

The DNC TG revealed no ECHO phenotype without the addition of stress from HAART that contained NRTIs. In AZT-containing HAART protocols, TG + AZT HAART mice increased normalized LV mass (mg/g body weight) to $1.39 \pm 0.10$ compared to $0.94 \pm 0.06$ in TG + vehicle. WT + AZT HAART LV mass was $0.85 \pm 0.04$ compared to $0.78 \pm 0.03$ in $\mathrm{WT}+$ vehicle. TG + AZT HAART LV mass was greater than that of any other in that experimental cohort $(P<0.001$; ANOVA; Figure 2a).

Analogous changes were seen with stavudinecontaining (D4T) HAART protocols. TG $+\mathrm{D} 4 \mathrm{~T}$ 

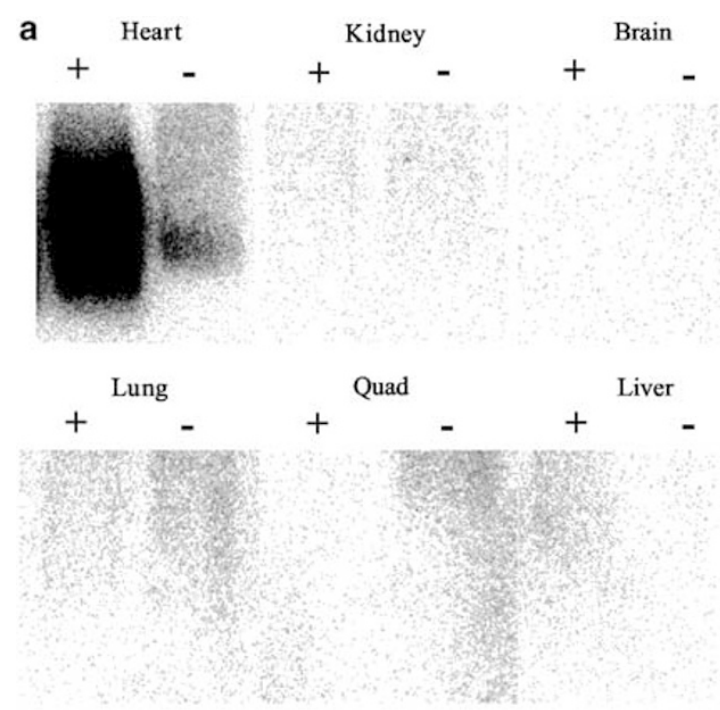

b
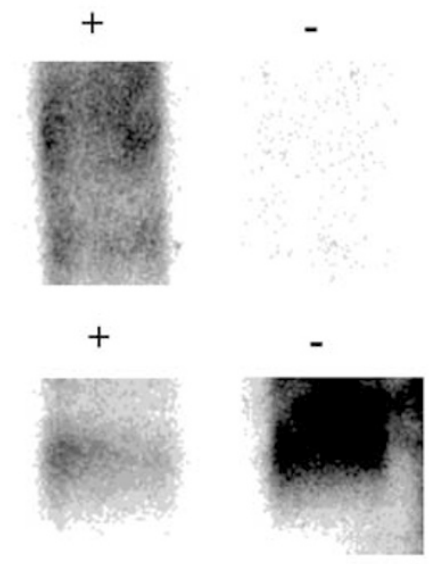

GAPDH

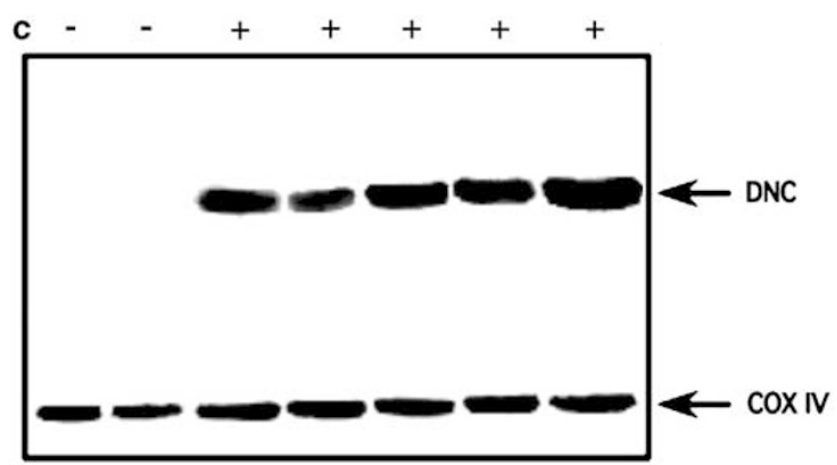

Figure 1 DNC TG analysis: (a) Northern analysis of TG with targeted DNC in heart: TG $(+)$ expression of DNC is significantly greater than WT (-) in heart. Expression in other tissues was similar in both TG and WT; (b) polyA ${ }^{+}$RNA from TG cardiac extracts was increased compared to WT (-) levels; (c) Western blot against DNC confirms abundant DNC polypeptide in TG mitochondrial extracts. Mitochondrial protein extracts were normalized using antiserum against subunit IV of cytochrome $c$ oxidase (COX IV).

HAART LV mass was $1.44 \pm 0.07$ compared to $1.08 \pm 0.08$ in $\mathrm{TG}+$ vehicle. $\mathrm{WT}+\mathrm{D} 4 \mathrm{~T}$ HAART $\mathrm{LV}$ mass was $0.95 \pm 0.06$ compared to $0.89 \pm 0.05$ in WT + vehicle. Again, the TG cohort treated with
HAART that contained NRTIs exhibited the largest LV mass in that experimental group $(P<0.001$; ANOVA; Figure 2b). In contrast to findings from these NRTI-containing HAART regimens, TGs and WT treated with the NRTI-sparing HAART regimen (nevirapine, efavirenz, indinavir) revealed no effect on cardiac LV mass (data not shown).

Cardiac MRI studies corroborated ECHO findings in TG + AZT HAART protocols. Representative images of both four chamber and biventricular plane sections (Figure 2c; paired figures) highlighted the structure of the ventricle walls and chamber cavities in two planes. TG + AZT HAART demonstrated cardiomegaly and increased ventricular volume. Quantitation of LV cavity area revealed a $19-22 \%$ increase in TG + AZT HAART LV compared to any other in that protocol.

\section{Ultrastructural Features of Mitochondria in TG Hearts}

The EM hallmark of cardiac targeted overexpression of DNC in TGs was found in mitochondria (Figure 3a and $b$ ). The striking and prevalent finding in DNC TG cardiac mitochondria was reduplicated cristae. This occurred in the absence of HAART treatment, but appeared to be more pronounced in TGs treated with HAART that contained NRTIs (see below). Treatment with HAART that contained NRTIs caused mitochondrial destruction and amplified damage from DNC overexpression. Features of NRTI-containing HAART treatment of DNC TGs included cardiac mitochondrial cristae reduplication (as seen in untreated TGs), but mitochondrial cristae dissolution (Figure $3 \mathrm{a}$ and $\mathrm{b}$, bottom right panels), and accumulation of intramitochondrial amorphous material (Figure 3a and b, arrows) were found with treatment. Together, these suggested worse damage and correlated with CM from HAART that contained NRTIs.

Quantitative analysis of mitochondrial damage was performed on EM of myocardial samples to confirm the pathological impressions (above). The proportion of damaged mitochondria was represented as a fraction of the total number of mitochondria per field. The proportion was determined in blinded examinations of myocardial profiles of samples from the treatment cohorts $(n \geq 187$ mitochondria per sample group). Results demonstrated a significant increase in the damaged mitochondria (Figure 3c; $P<0.01$; ANOVA) in myocardial samples from DNC TGs treated with NRTI-containing HAART (approximately $40 \%$ damaged mitochondria; more than in any other cohort; Figure 3c) and confirmed impressions from the ultrastructural profiles.

\section{Real-Time ECG and Plasma Lactate}

DNC and WT littermates with and without HAART $(N=4-11)$ were evaluated by ECG. Both NRTI-con- 
976
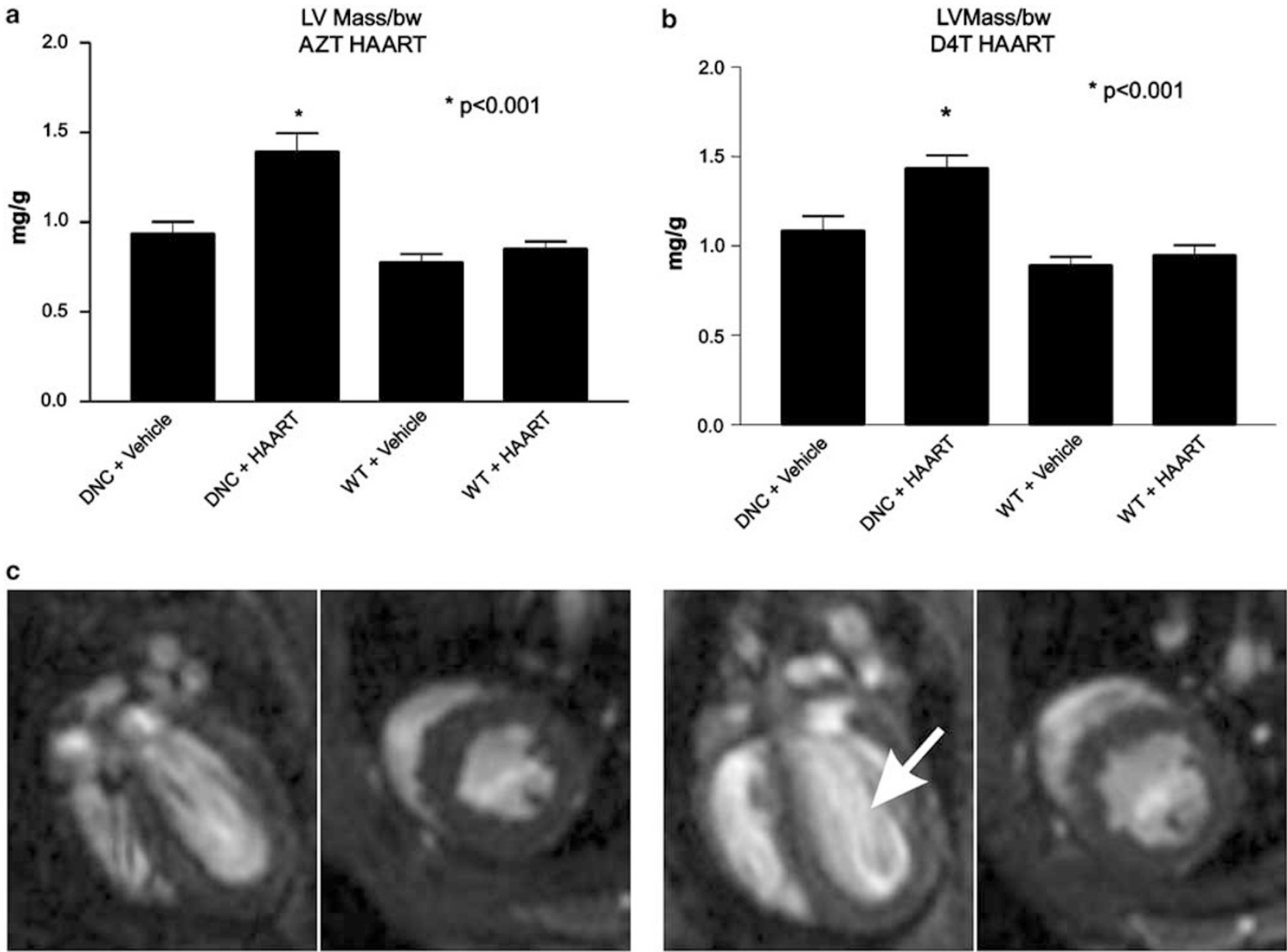

$\mathrm{TG}+\mathrm{CMC}$
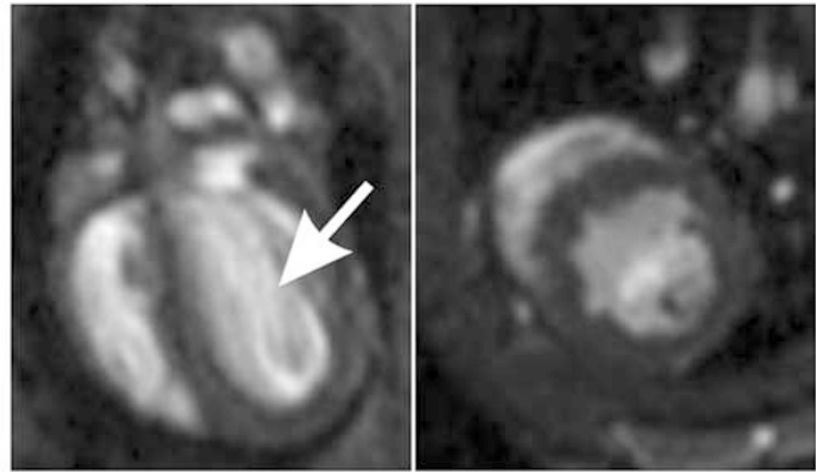

TG+ AZT HAART
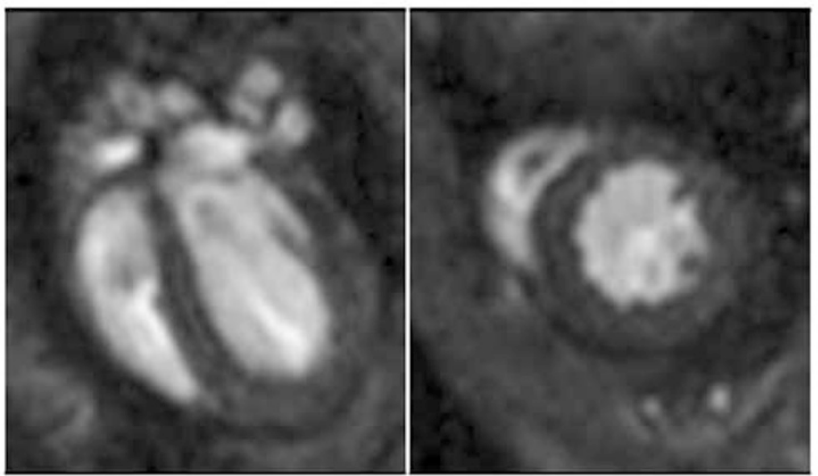

WT+CMC
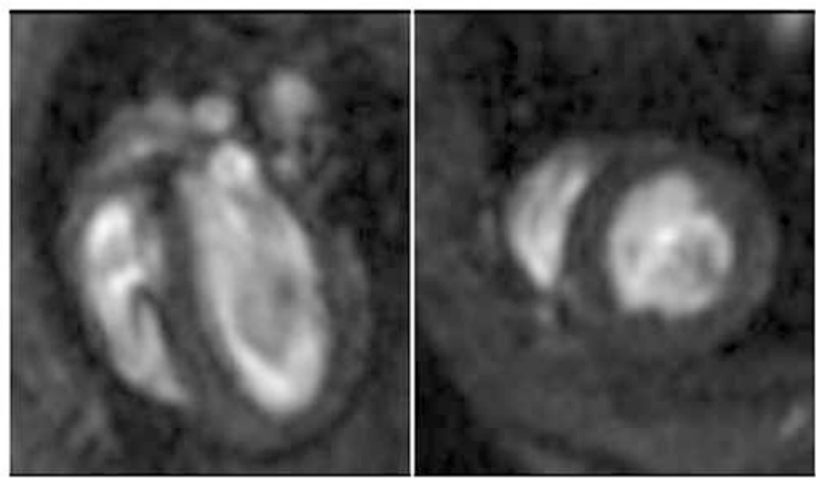

WT+AZT HAART

Figure 2 Quantitative analysis of ECHO images (a and b) and MRI images (c): histograms of calculated LV mass in treatment cohorts from HAART containing AZT (a),and HAART containing D4T (b). LV mass was calculated in a blinded manner, code was broken, and data tabulated. Data are normalized to body weight $(\mathrm{mg} / \mathrm{g})$ and plotted as mean \pm s.e.m. In either TG + HAART protocol, the TG cohort treated with HAART revealed profoundly increased LV mass after 35 days $(P<0.05$; ANOVA). (c) MRI long-axis (left of pairs) and short-axis (biventricular) views of hearts from TG and WT mice treated with HAART: Cohorts were treated with HAART containing AZT backbone. TG + AZT HAART reveals cardiomegaly and increased ventricular chamber dimensions, particularly the LV (arrow).

taining and NRTI-sparing cohorts were examined. HRV was the principal abnormality and was found only in HAART-treated TG cohorts (Figure 4a and b; $P<0.05$; ANOVA) irrespective of the NRTI used. No change in HRV was found in cohorts treated with
NRTI-sparing HAART regimens. After 35 days (Figure 4c), TG + AZT HAART had elevated plasma lactate $(3.24 \pm 0.39 \mathrm{mM})$ compared to all other cohorts in that protocol $(P<0.05$; ANOVA $)$, but an analogous change was neither found with D4T-based 

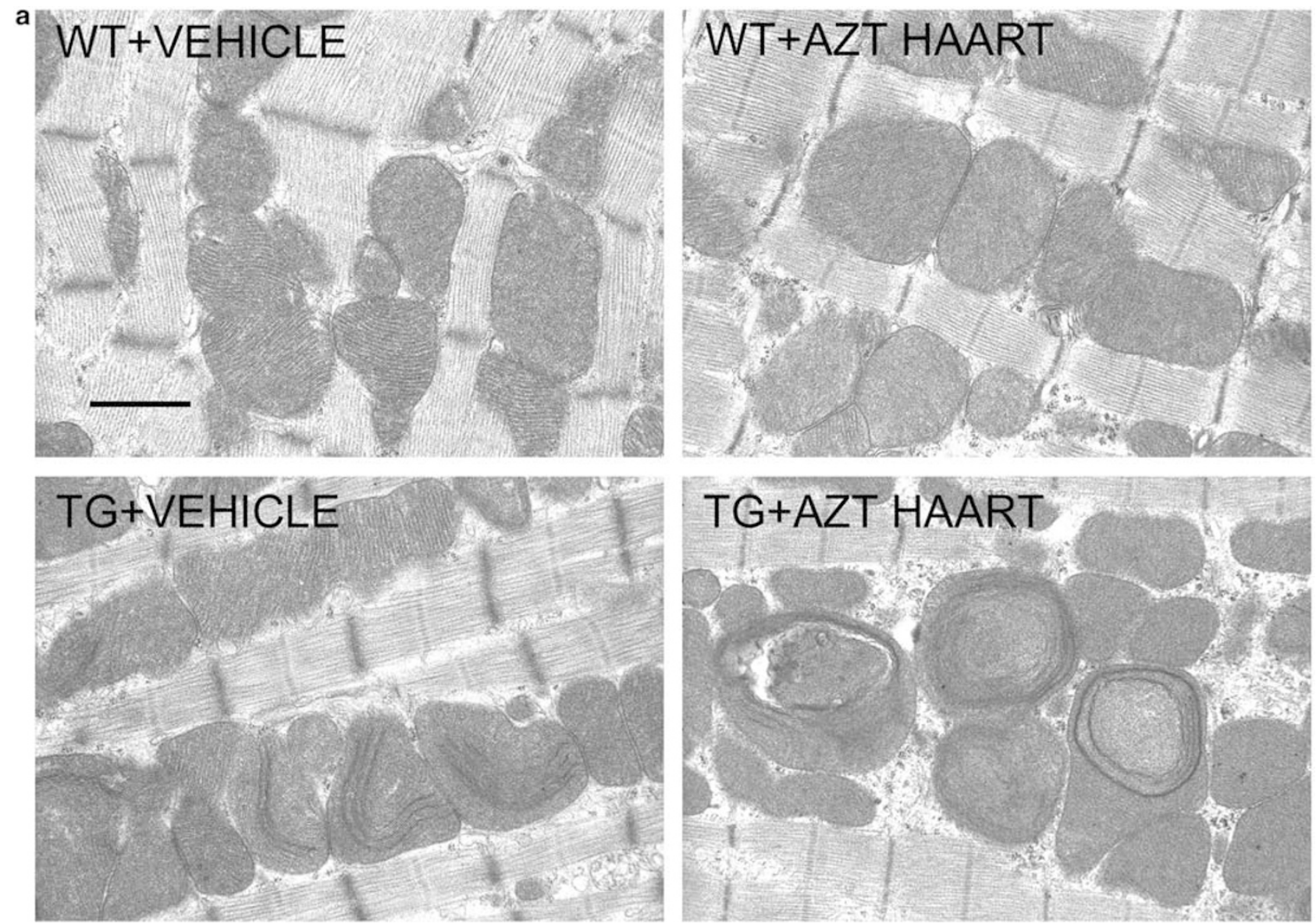

Figure 3 Electron microscopic (a and b) views of mitochondria from TG and WT hearts and quantitative analysis (c): DNC overexpression in the heart caused mitochondrial cristae reduplication (top left, a and b). Addition of HAART containing NRTIs (either AZT/3TC/indinavir; or D4T/3TC/indinavir; 35 days) resulted in mitochondrial destruction, loss of cristae, and amorphous deposits (arrow, bottom right panel (a and b), original magnification on EM: 26 000; marker indicates $1 \mu$ ). Morphometric analysis confirmed increased mitochondrial damage in cardiac mitochondria from HAART-treated TGs $(\mathbf{c} ; P<0.01$; ANOVA)

HAART nor with NRTI-sparing HAART regimens (not shown).

\section{Discussion}

The phenotype that resulted from targeted myocardial DNC overexpression was relevant to cardiac mitochondrial ultrastructure, mitochondrial dysfunction, and to AIDS therapy. NRTIs are fundamental components of HAART, but mitochondrial toxicity to different tissue targets is a therapeutic obstacle. ${ }^{4}$ In this study, untreated DNC TGs exhibited structural mitochondrial changes on EM, but no dysfunction. Mitochondrial changes were amplified and accompanied cardiac dysfunction in cohorts treated with HAART that contained NRTIs. In contrast, NRTI-sparing regimens caused no functional changes. The cellular processes of NRTI mitochondrial import and phosphorylation ${ }^{16-25}$ received increasing interest and awareness as playing a role in the regulation of mtDNA replication. ${ }^{17}$ The
DNC TG here offered an approach to evaluate NRTI toxicity in vivo and linked NRTI toxicity to mitochondrial import and nucleotide homeostasis. ${ }^{4}$

For some time, our group ${ }^{26-28}$ and others ${ }^{29-35}$ have studied mechanisms of mitochondrial toxicity of NRTIs focusing on inhibition of DNA pol $\gamma$ (reviewed in Kaguni ${ }^{36}$ ). NRTIs are firmly linked to altered mtDNA replication ${ }^{10,37,38}$ through the $D N A$ pol $\gamma$ hypothesis. ${ }^{39}$ This was expanded to include oxidative stress and mtDNA mutations in a 'mitochondrial dysfunction hypothesis',40 where the importance of intramitochondrial availability of NRTIs and other features were increasingly apparent. Data herein in vivo underscore points of the hypotheses.

Previous in vivo studies with TGs and/or NRTIs focused on the impact of HIV as an independent variable for morbidity. ${ }^{9,41-46}$ It is reasonable to conclude that DNC plays a mechanistic role in mitochondrial defects from thymidine analog-based HAART. Intramitochondrial abundance of phosphorylated and unphosphorylated native nucleo- 

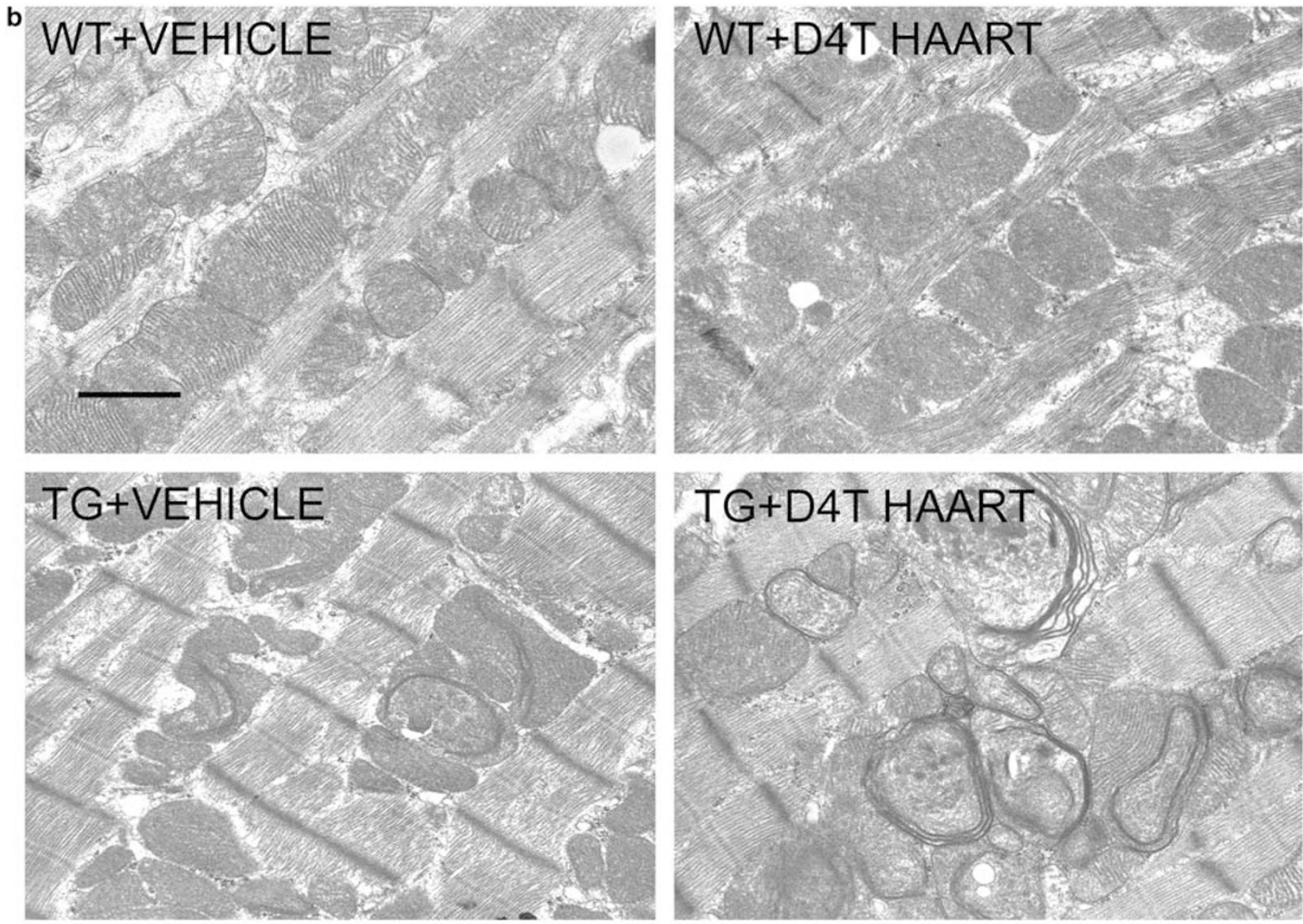

c

\section{Morphometric Analysis of} Mitochondrial Damage

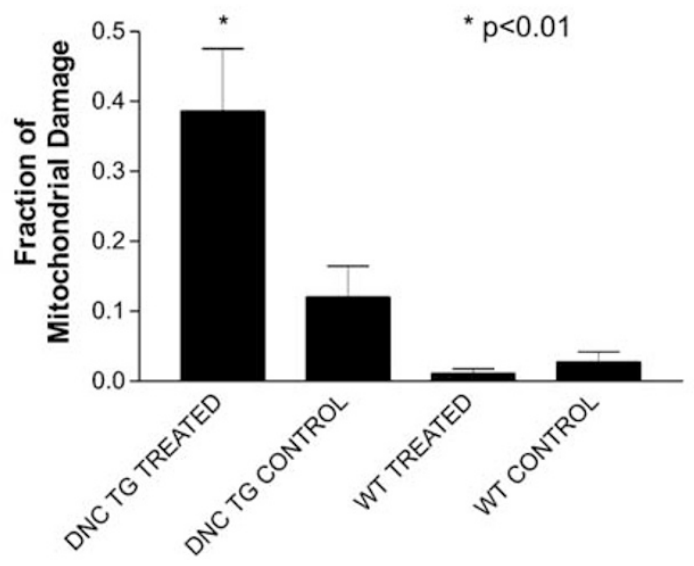

Figure 3 Continued.

sides and NRTIs affects inhibition mtDNA replication at the level of the nucleotide substrate. The DNC TG helps explain compartmentalization and utilization of NRTIs in mitochondria. Moreover, the absence of CM changes in TG and WT cohorts treated with an NRTI-sparing HAART emphasizes the importance of NRTIs in the pathophysiology of deleterious mitochondrial events.
Energy deprivation in other $\mathrm{CMs}^{47,48}$ may be critical to mitochondrial dysfunction. The initiating step of NRTI toxicity here may be mtDNA depletion, decreased energy abundance, or structurally abnormal mitochondria. Energy deprivation was absent basally in DNC TGs. Treatment with HAART that contained NRTIs resulted in full-blown CM. In contrast, CM was absent with NRTI-sparing HAART 
a

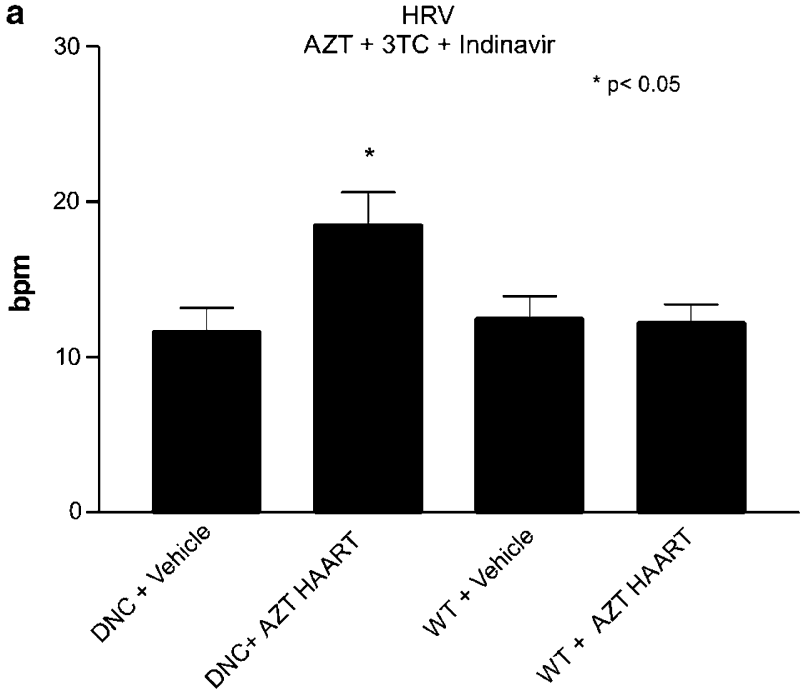

b

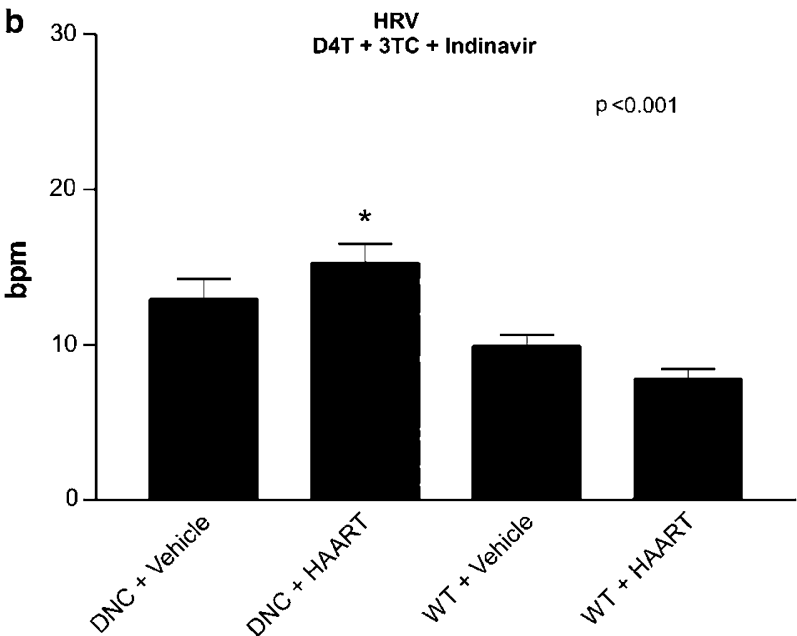

c
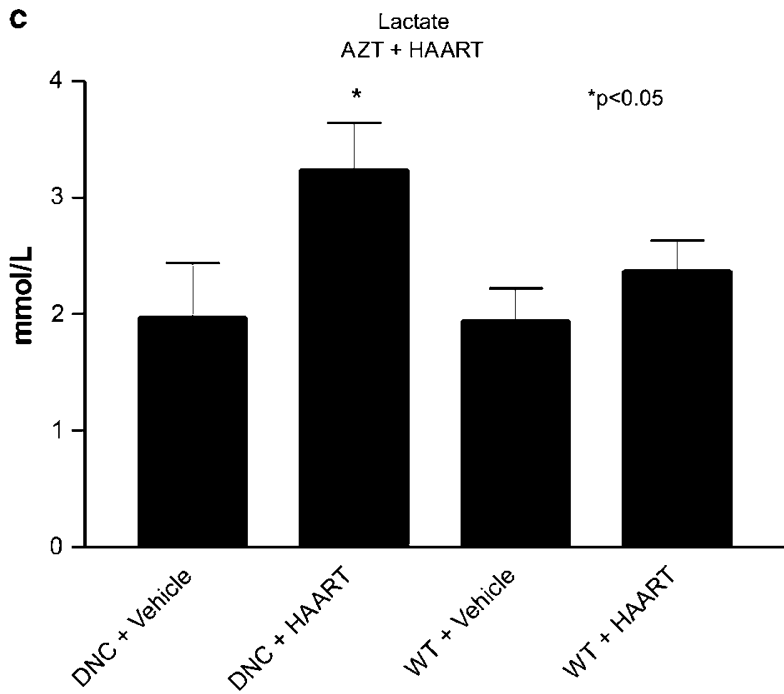

Figure 4 ECG data (a and b) and plasma lactate (c) from HAART treatment protocols: Quantitative data from real-time ECG revealed significant HRV in TG + HAART with AZT (a) or with D4T (b) backbone. Samples from the TG + AZT-based HAART revealed elevated plasma lactate compared to any other cohort (c; $P<0.05 ;$ ANOVA). treatment. It remains to be determined whether the primary mechanism in DNC TGs is increased intramitochondrial NRTI concentration, disruption of nucleotide homeostasis (and mtDNA replication), or if other mechanisms are involved. In the studies herein, a time course of events that lead to the end point were not undertaken.

Ultrastructural features were key findings in DNC TG hearts and were corroborated morphometrically. Mitochondrial ultrastructural defects were amplified by HAART. This point was confirmed quantitatively. The ultrastructural features of TGs treated with either AZT- or D4T-containing HAART were similar.

It may be worthwhile to speculate that DNC plays a role in AIDS CM. ${ }^{49}$ Samples from fetal primates treated with NRTIs in utero showed cardiac mitochondrial structural defects. ${ }^{50}$ mtDNA mutations were found in tissue samples after cessation of NRTIs received in utero. ${ }^{51}$ Since CM occurred with AZT- and D4T-based HAART, both NRTIs were functionally and structurally toxic to mitochondria.

In summary, we expressed human DNC transgenically in the murine heart. Cardiac mitochondria from these DNC TG hearts had reduplicated cristae and amorphous deposits, but no dysfunction. CM occurred in TGs treated with HAART that contained NRTIs. CM features included increased LV mass, LV dilation, increased HRV, hyperlactatemia and mitochondrial destruction. HAART regimens without NRTIs caused no changes of CM. Future studies with DNC TGs may help further clarify mechanisms of NRTI toxicity to mitochondria and how the effects may be prevented or ameliorated in patients.

\section{Acknowledgements}

This work was supported by DHHS, NIH NHLBI R01 HL072707 and HL059798 to WL and by MIUR-PRIN, CEGBA and the European Community's Sixth Framework Programme for Research, Priority 1 'Life sciences, genomics and biotechnology for health', contract number LSHM-CT-2004-503116 to FP.

\section{References}

1 Palmieri F. The mitochondrial transporter family (SLC25): physiological and pathological implications. Pflugers Archiv - Eur J Physiol 2004;447:689-709.

2 Dolce V, Fiermonte G, Runswick MJ, et al. The human mitochondrial deoxynucleotide carrier and its role in the toxicity of nucleoside antivirals. Proc Natl Acad Sci USA 2001;98:2284-2288.

3 Palmieri F. Mitochondrial carrier proteins. FEBS Lett 1994;346:48-54.

4 Lewis W, Day BJ, Copeland WC. Mitochondrial toxicity of NRTI antiviral drugs: an integrated cellular perspective. Nat Rev Drug Discov 2003;2:812-822.

5 Joly V, Descamps D, Yeni P. NNRTI plus PI combinations in the perspective of nucleoside-sparing or nucleoside-failing antiretroviral regimens. AIDS Rev 2002;4:128-139. 
6 Raidel SM, Haase C, Jansen NR, et al. Targeted myocardial transgenic expression of HIV Tat causes cardiomyopathy and mitochondrial damage. Am J Physiol Heart Circ Physiol 2002;282:H1672-H1678.

7 Subramaniam A, Jones WK, Gulick J, et al. Tissuespecific regulation of the alpha-myosin heavy chain gene promoter in transgenic mice. J Biol Chem 1991; 266:24613-24620.

8 Lewis W, Haase CP, Raidel SM, et al. Combined antiretroviral therapy causes cardiomyopathy and elevates plasma lactate in transgenic AIDS mice. Lab Invest 2001;81:1527-1536.

9 Lewis W, Grupp IL, Grupp G, et al. Cardiac dysfunction occurs in the HIV-1 transgenic mouse treated with zidovudine. Lab Invest 2000;80:187-197.

10 Dalakas MC, Illa I, Pezeshkpour GH, et al. Mitochondrial myopathy caused by long-term zidovudine therapy [see comments]. N Engl J Med 1990;322: 1098-1105.

11 Sutliff RL, Haase C, Russ R, et al. Cocaine increases mortality and cardiac mass in a murine transgenic model of acquired immune deficiency syndrome. Lab Invest 2003;83:983-989.

12 Chu V, Otero JM, Lopez O, et al. Electrocardiographic findings in mdx mice: a cardiac phenotype of Duchenne muscular dystrophy. Muscle Nerve 2002;26: 513-519.

13 Chu V, Otero JM, Lopez O, et al. Method for noninvasively recording electrocardiograms in conscious mice. BMC Physiol 2001;1:6.

14 Mitchell GF, Jeron A, Koren G. Measurement of heart rate and $\mathrm{Q}-\mathrm{T}$ interval in the conscious mouse. Am J Physiol 1998;274:H747-H751.

15 Gehrmann J, Hammer PE, Maguire CT, et al. Phenotypic screening for heart rate variability in the mouse. Am J Physiol Heart Circ Physiol 2000;279:H733-H740.

16 Rinaldo-Matthis A, Rampazzo C, Balzarini J, et al. Crystal structures of the mitochondrial deoxyribonucleotidase in complex with two specific inhibitors. Mol Pharmacol 2004;65:860-867.

17 Rampazzo C, Ferraro P, Pontarin G, et al. Mitochondrial deoxyribonucleotides, pool sizes, synthesis, and regulation. J Biol Chem 2004;279:17019-17026.

18 Mazzon C, Rampazzo C, Scaini MC, et al. Cytosolic and mitochondrial deoxyribonucleotidases: activity with substrate analogs, inhibitors and implications for therapy. Biochem Pharmacol 2003;66:471-479.

19 Rampazzo C, Gallinaro L, Milanesi E, et al. A deoxyribonucleotidase in mitochondria: involvement in regulation of $\mathrm{dNTP}$ pools and possible link to genetic disease. Proc Natl Acad Sci USA 2000; 97:8239-8244.

20 Wang L, Saada A, Eriksson S. Kinetic properties of mutant human thymidine kinase 2 suggest a mechanism for mitochondrial DNA depletion myopathy. J Biol Chem 2003;278:6963-6968.

21 Saada A, Ben-Shalom E, Zyslin R, et al. Mitochondrial deoxyribonucleoside triphosphate pools in thymidine kinase 2 deficiency. Biochem Biophys Res Commun 2003;310:963-966.

22 Saada A, Shaag A, Elpeleg O. mtDNA depletion myopathy: elucidation of the tissue specificity in the mitochondrial thymidine kinase (TK2) deficiency. Mol Genet Metab 2003;79:1-5.

23 Dobrovolsky VN, Bucci T, Heflich RH, et al. Mice deficient for cytosolic thymidine kinase gene develop fatal kidney disease. Mol Genet Metab 2003;78:1-10.
24 Saada A, Shaag A, Mandel H, et al. Mutant mitochondrial thymidine kinase in mitochondrial DNA depletion myopathy. Nat Genet 2001;29:342-344.

25 Wang J, Su C, Neuhard J, et al. Expression of human mitochondrial thymidine kinase in Escherichia coli: correlation between the enzymatic activity of pyrimidine nucleoside analogues and their inhibitory effect on bacterial growth. Biochem Pharmacol 2000;59: 1583-1588.

26 Lewis W, Papoian T, Gonzalez B, et al. Mitochondrial ultrastructural and molecular changes induced by zidovudine in rat hearts. Lab Invest 1991;65:228-236.

27 Lewis W, Simpson JF, Meyer RR. Cardiac mitochondrial DNA polymerase-gamma is inhibited competitively and noncompetitively by phosphorylated zidovudine. Circ Res 1994;74:344-348.

28 Lewis W, Levine ES, Griniuviene B, et al. Fialuridine and its metabolites inhibit DNA polymerase gamma at sites of multiple adjacent analog incorporation, decrease mtDNA abundance, and cause mitochondrial structural defects in cultured hepatoblasts. Proc Natl Acad Sci USA 1996;93:3592-3597.

29 Lee H, Hanes J, Johnson KA. Toxicity of nucleoside analogues used to treat AIDS and the selectivity of the mitochondrial DNA polymerase. Biochemistry 2003; 42:14711-14719.

30 Feng JY, Murakami E, Zorca SM, et al. Relationship between antiviral activity and host toxicity: comparison of the incorporation efficiencies of $2^{\prime}, 3^{\prime}$-dideoxy-5fluoro-3'-thiacytidine-triphosphate analogs by human immunodeficiency virus type 1 reverse transcriptase and human mitochondrial DNA polymerase. Antimicrob Agents Chemother 2004;48:1300-1306.

31 Johnson AA, Ray AS, Hanes J, et al. Toxicity of antiviral nucleoside analogs and the human mitochondrial DNA polymerase. J Biol Chem 2001;276:40847-40857.

32 Feng JY, Johnson AA, Johnson KA, et al. Insights into the molecular mechanism of mitochondrial toxicity by AIDS drugs. J Biol Chem 2001;276:23832-23837.

33 Graziewicz MA, Sayer JM, Jerina DM, et al. Nucleotide incorporation by human DNA polymerase gamma opposite benzo[a]pyrene and benzo[c]phenanthrene diol epoxide adducts of deoxyguanosine and deoxyadenosine. Nucleic Acids Res 2004;32:397-405.

34 Copeland WC, Ponamarev MV, Nguyen D, et al. Mutations in DNA polymerase gamma cause error prone DNA synthesis in human mitochondrial disorders. Acta Biochim Pol 2003;50:155-167.

35 Lim SE, Copeland WC. Differential incorporation and removal of antiviral deoxynucleotides by human DNA polymerase gamma. J Biol Chem 2001;276: 23616-23623.

36 Kaguni LS. DNA polymerase gamma, the mitochondrial replicase 1. Annu Rev Biochem 2004;73:293-320.

37 Swartz MN. Mitochondrial toxicity-new adverse drug effects [editorial; comment]. N Engl J Med 1995; 333:1099-1105.

38 Arnaudo E, Dalakas M, Shanske S, et al. Depletion of muscle mitochondrial DNA in AIDS patients with zidovudine-induced myopathy. Lancet 1991;337: 508-510.

39 Lewis W, Dalakas MC. Mitochondrial toxicity of antiviral drugs. Nat Med 1995;1:417-422.

40 Lewis W, Copeland WC, Day B. Mitochondrial DNA depletion, oxidative stress and mutation: mechanisms of nucleoside reverse transcriptase inhibitor toxicity. Lab Invest 2001;81:777-790. 
41 Ray PE, Liu XH, Robinson LR, et al. A novel HIV-1 transgenic rat model of childhood HIV-1-associated nephropathy. Kidney Int 2003;63:2242-2253.

42 Reid W, Sadowska M, Denaro F, et al. An HIV-1 transgenic rat that develops HIV-related pathology and immunologic dysfunction. Proc Natl Acad Sci USA 2001;98:9271-9276.

43 Hanna Z, Weng X, Kay DG, et al. The pathogenicity of human immunodeficiency virus (HIV) type 1 nef in cd4c/HIV transgenic mice is abolished by mutation of its sh3-binding domain, and disease development is delayed in the absence of hck. J Virol 2001;75:93789392.

44 Dickie P. Nef modulation of HIV type 1 gene expression and cytopathicity in tissues of HIV transgenic mice. AIDS Res Hum Retroviruses 2000;16:777-790.

45 Prakash O, Joshi BH, Zhang P, et al. Transgenic mouse model of ethanol as a cofactor in HIV disease. Alcohol Clin Exp Res 1998;22:266S-268S.
46 Tinkle BT, Ueda H, Ngo L, et al. Transgenic dissection of HIV genes involved in lymphoid depletion. J Clin Invest 1997;100:32-39.

47 Katz AM. Is the failing heart energy depleted? Cardiol Clin 1998;16:633-644, viii.

48 Katz AS, Sadaniantz A. Echocardiography in HIV cardiac disease. Prog Cardiovasc Dis 2003;45:285-292.

49 Lewis W. Defective mitochondrial DNA replication and NRTIs: pathophysiological implications in AIDS cardiomyopathy. Am J Physiol Heart Circ Physiol 2003;284:H1-H9.

50 Gerschenson M, Nguyen V, Ewings EL, et al. Mitochondrial toxicity in fetal Erythrocebus patas monkeys exposed transplacentally to zidovudine plus lamivudine. AIDS Res Hum Retroviruses 2004;20:91-100.

51 Divi RL, Walker VE, Wade NA, et al. Mitochondrial damage and DNA depletion in cord blood and umbilical cord from infants exposed in utero to Combivir. AIDS 2004;18:1013-1021. 